\title{
Virtual Dressing Room Application with Virtual Human Using Kinect Sensor
}

\author{
Muhammed Kotan ${ }^{1}$ and Cemil Öz ${ }^{2}$ \\ 1. Department of Information Systems Engineering, Faculty of Computer and Information Sciences, Sakarya University, Sakarya \\ 54187, Turkey \\ 2. Department of Computer Engineering, Faculty of Computer and Information Sciences, Sakarya University, Sakarya 54187, Turkey
}

Received: March 24, 2015 / Accepted: April 15, 2015 / Published: May 25, 2015.

\begin{abstract}
This study proposes a real time 3D virtual model controll and a virtual dressing room application to enable users to try virtual garments and shoes on in front of a virtual mirror. A virtual representation of the user appears in a virtual changing room and the user's hand motions select the clothes from a list on the screen. Afterwards, the selected virtual clothes appear on a humanoid model in the virtual mirror. For the purpose of aligning the 3D garments and shoes with the model, 3D locations of the joints are used for positioning, scaling and rotating. By using our developed algorithm, small, medium, large or xlarge garment size is selected automatically and this information is shown on the screen. Then, we apply skin color detection to handle the unwanted occlusions between the user and the model. To create a more realistic effect, the system takes into account different images of the clothes according to different human poses and movements. Optional mirror selection buttons make it possible to have multiple viewing angles on the model. Additionally, we developed an algorithm for matching up all motions between the model and garments. In this study, we benefit from the Microsoft Kinect SDK (software development kit) in order to follow the user's movements, coordinate the suitable clothe try-ons and provide depth sort effect to the human body and clothes. In order to use the rapid calculation attributes of game engines, we used unity 3D game engine.
\end{abstract}

Key words: Virtual mirror, virtual try-on, virtual reality, virtual dressing room, Kinect for windows, human-computer interaction.

\section{Introduction}

Previously, people used to spend a lot of time while shopping. One could not see whether or not the clothes would fit without having to take off his or her own clothes to try out the new ones, and had to wait in a long queue outside the fitting rooms.

Virtual mirror projects provide support for online shopping by offering the facility of selected clothes try-on. Therefore, users can see how they look in the clothes without physically putting them on, and without spending a lot time. OpenNI organization develops an open-source, cross platform framework to process the data from a Kinect sensor. Microsoft Research has also released the Kinect SDK (software

Corresponding author: Muhammed Kotan, Res. Asst., M.Sc., research field: virtual reality, modeling, simulation and control systems. E-mail: mkotan@sakarya.edu.tr. development kit) a robust real time skeletal body tracker. Different virtual mirror projects have been in progress recently. For example, Givonni et al. [1] presents a virtual try-on system, which allows performance comparisons of their system with two skeletal tracking SDKs: OpenNI and Kinect for Windows SDK. Zhou et al. [2] proposes a real time approach for virtual clothes fitting using Kinect. Murata et al. [3] focuses on the "video mirror interface”. A user can operate a computer system by selecting virtual objects on a screen with his/her hand.

After its launch in 2010, Microsoft Kinect has become the state of the art depth image sensor in the market. There is currently a quite intensive study to implement application programming interfaces for developers including a skeletal body tracking method. We preferred to use the Microsoft Research Kinect 
SDK due to its robust and practical skeletal tracking algorithm.

Our approach can be summarized as follows:

- Positioning the 3D garments on a virtual dressing room;

- Scaling of the humanoid model by using received inputs from sensors;

- Positioning of the selected 3D cloth models on the humanoid model;

- Skin colour and gender detection;

- Using mirrors in order to have multiple viewing angles on the model.

The user interface allows the user to choose a garment by making a hand movement towards to it. A screenshot of the application is shown in Fig. 2.

The virtual mirror project presented in this paper has been developed mainly to help online shopping. In this work, we used the Microsoft Kinect for Windows sensor and the Kinect SDK. When the user moves freely in front of the mirror, the model and clothes follow accordingly to such movements.

The rest of the paper is organized as follows: Section 2 presents the creation of whole 3D models used in the application; Section 3 discusses the implementation of the virtual mirror system; and Section 4 gives the conclusions.
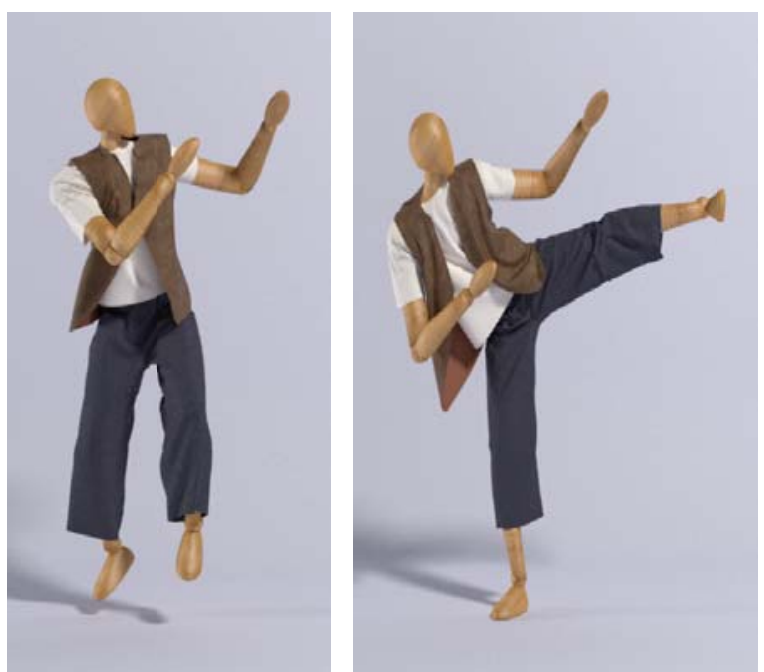

Fig. 1 The figures about physics based simulation for clothing obtained from a recent study [4].

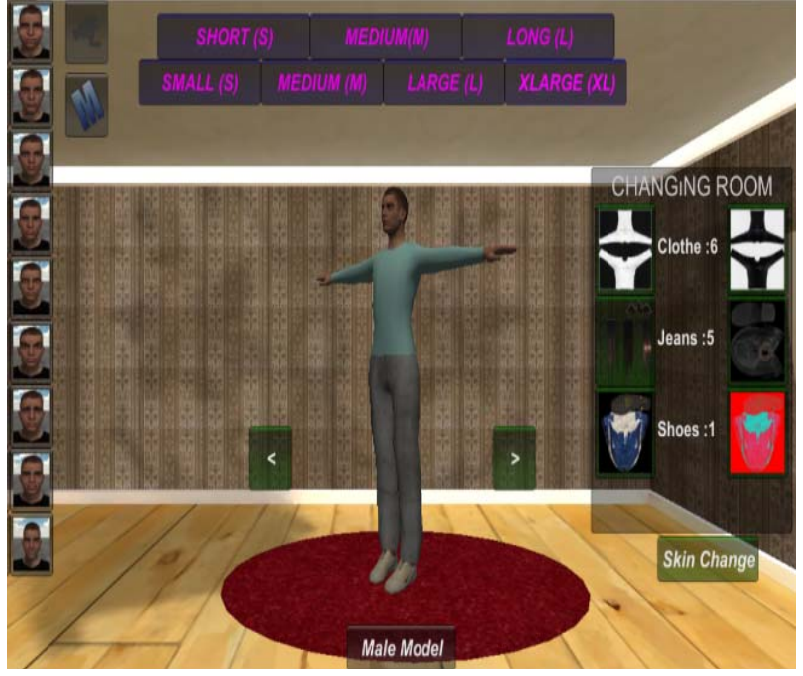

Fig. 2 The user interface of the application.

\section{Creating Models}

Computer graphics gives us the power to model and animate virtual humans. To simulate humans requires real-time visualization and animation, taking into account constraints on the data used for these avatars (virtual humans representing users).

In literature, several approaches are proposed for skeletal tracking and body part detection. For example, Shotton et al. [5] have developed a system that predicts the 3D positions of body joints from a single depth image. Kjærside et al. [6] propose a tag-based approach which requires manual labeling of body parts in order to create an augmented reality of the customer wearing a cloth simulation.

\subsection{Humanoid Models}

In this study, two basic models including a man and a woman are created in MakeHuman program-an Open Source software (AGPL3.0) for creating lightweight, realistic 3D models of the human form. MakeHuman $^{\mathrm{TM}}$ is a tool designed to simplify the creation of virtual humans using a GUI (graphical user interface), also commonly referred to as a GUI. This is a specialized branch of the more general subject of 3D modeling [7]. A sample rendered male model is shown in Fig. 4. 


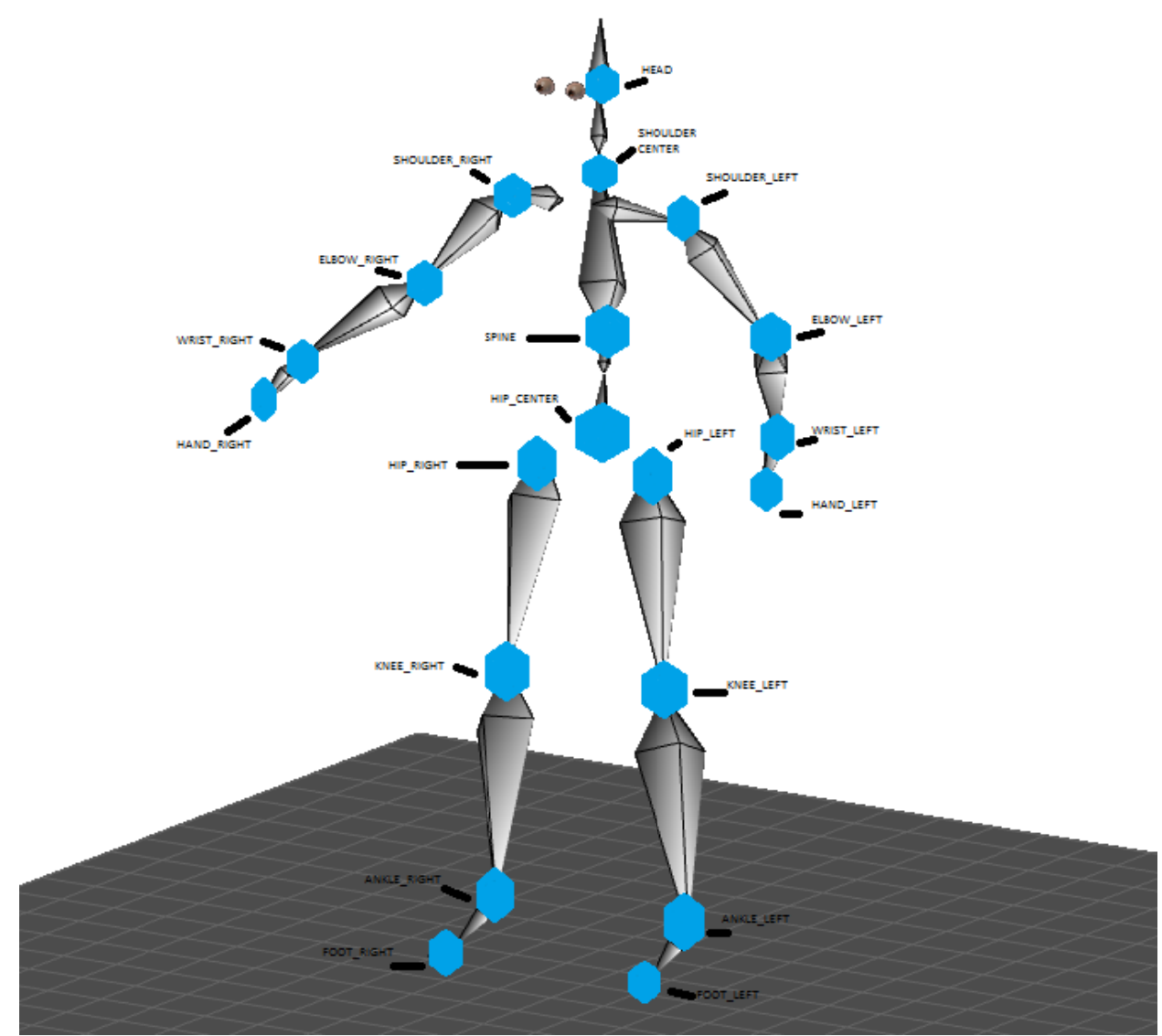

Fig. 3 Our skeleton joint structure for the avatars.

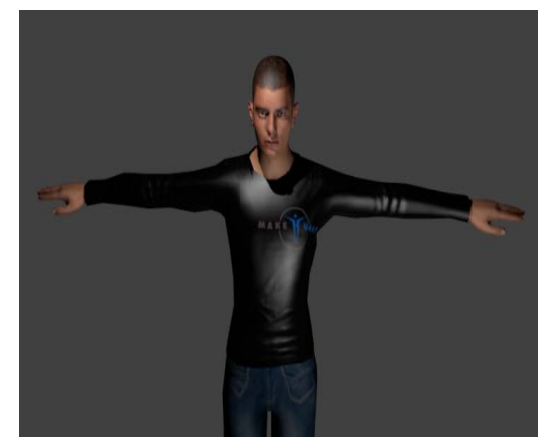

Fig. 4 Rendered male model in Blender 3D v2.69.

In addition to their parametric and flexible parts, models have more than 200 joints. At the present ready-made clothing, body size is standardized and named as small, medium, large, xlarge, etc. Because a size medium can differ from one brand to another, the better scenario is to see the clothes on one's own body.

The body size variations of our models were generated in the order of standardized body sizes. Skin colors of the models have parametric structures, so that preferred skin color can be selected as white, dark, etc. Since the most important parts of the models are their face, some facial expressions like smiling, crying, serious, sad, etc. included, and they all were designed for both male and female models. Fig. 6 illustrates sample expressions for the humanoid models.

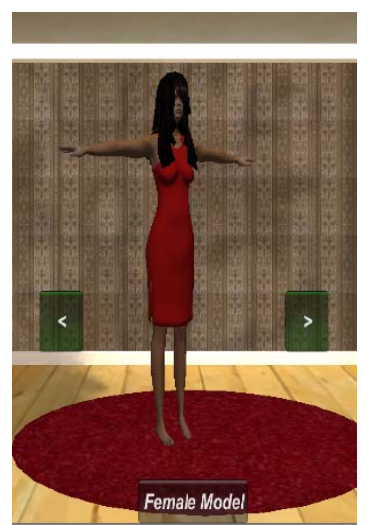

(a)

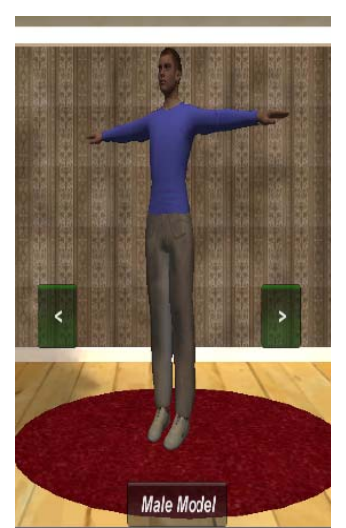

(b)
Fig. 5 Female (a) and male (b) humanoid models form the GUI of the application created in unity $3 D$. 


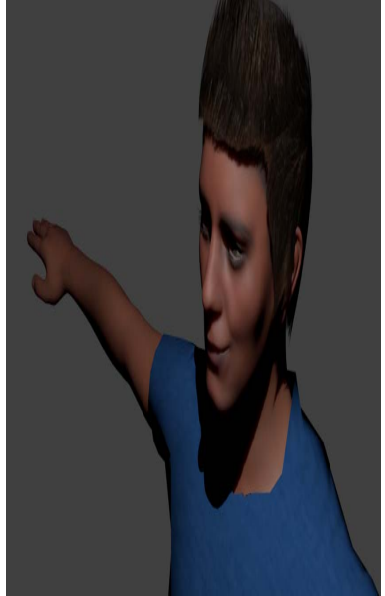

(a)

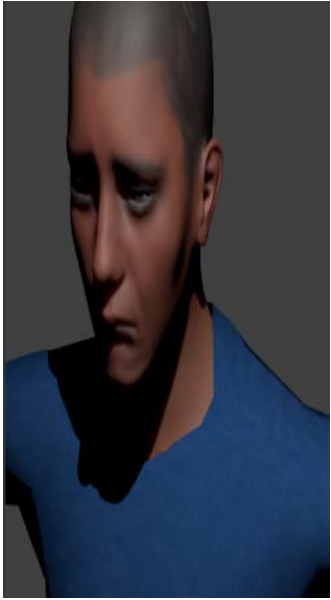

(b)
Fig. 6 Happy (a) and sad (b) models are being rendered, recursively.

\subsection{Garment and Shoe Models}

T-shirts, cardigans, jackets, dress, trousers, and skirts are some of modeled upper and lower body garments. An additional model has been created to take 3D clothe models produced for standard clothing automatically. The shoes are modeled separately.

Virtual mirror projects are also used for glasses, jewelries, handbags, etc. For example, Cho et al. [8] developed a system in which the user is able to try on glasses. Wang et al. [9] developed an augmented reality system for online shopping in which users can experiment with virtual handbags in different ways.

\subsection{Virtual Changing Room and Components}

To try virtual garments and shoes on in front of a virtual mirror, we created and designed virtual rooms similar to changing-rooms in clothing stores. Alternatively, we generated optional mirror select buttons for a backward vision of garments and shoes.

\section{Performing the Virtual Mirror System}

Game engines are widely used to ensure interaction with the virtual world especially in games, virtual reality systems and simulations. Without using game engines, creation of scenes, exporting models, running readable input units and reshaping the scene according to inputs and performing possible physics and dynamics of the event require a long time and comprehensive programming knowledge. That is why the unity game engine is used in this study. Unity is a game development ecosystem: a powerful rendering engine fully integrated with a complete set of intuitive tools and rapid workflows to create interactive 3D and 2D content; easy multiplatform publishing; thousands of quality, ready-made assets in the asset store and a knowledge-sharing community [10].

Figs. 2 and 5 are created in Unity 3D. User input unit can be one of the input devices like keyboard, mouse, web-cam or Microsoft Kinect. The interaction with created models is possible by these units. If the user has a web-cam or Kinect, the system can determine the user's gender, height and body size automatically. Also, they can be chosen manually with a keyboard or mouse. Moreover, it is possible to manually or automatically rotate the model by the use of input units. There is also a clothe panel that enable users to change garments and shoes, and their colors or patterns as wished.

Skin color: skin color is an important and essential parameter in clothing. One desires to wear dresses compatible with the skin color he or she has. Skin color is selectable by users in virtual interface and can be determined automatically with a webcam or Kinect. With the help of computer vision methods and openCV program, the model's skin color is set to a specific color obtained from user's own skin. Fig. 7 illustrates an example for skin color and emotional detection of user based model.

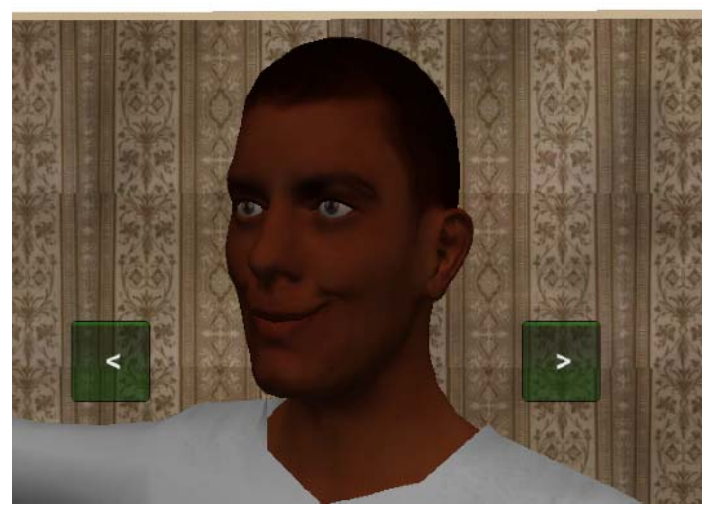

Fig. 7 Skin color detection. 


\section{Conclusions}

In this study, a virtual mirror system is designed for the purpose of clothe changing room. Our motivation here is to increase the time efficiency and improve the accessibility of clothes try-on by creating a virtual dressing room environment.

The system contains two basic male and female models of which joints are over 200. They have spectacular potential of performing human movements as well as facial expressions. For the body size and height, we created a lot of model variations. The GUI of changing-room reads and interprets the data arrived form keyboard, mouse, webcam or Kinect input units and enables users to try garments and shoes on a created humanoid model.

\section{References}

[1] Giovanni, S., Choi, Y. C., Huang, J., Khoo, E. T., and Yin, K. 2012. "Virtual Try-on Using Kinect and HD Camera.” In Proceedings of MIG 2012, 55-65.

[2] Zhou, Z., Shu, B., Zhuo, S., Deng, X., Tan, P., and Lin, D. 2012. "Image-based Clothes Animation for Virtual Fitting.” Presented at the SIGGRAPH Asia 2012 Technical Briefs, Singapore.
[3] Murata, K., Hattori, M., and Shibuya, Y. 2013. "Effect of Unresponsive Time for User's Touch Action of Selecting an Icon on the Video Mirror Interface." HCI International 8007: 462-8.

[4] Narain, R., Samii, A., and O’Brien, J. F. 2012. “Adaptive Anisotropic Remeshing for Cloth Simulation.” ACM Transactions on Graphics 31 (6): Article No.152.

[5] Shotton, J., Fitzgibbon, A., Cook, M., Sharp, T., Finocchio, M., Moore, R., Kipman, A., and Blake, A. 2011. "Real-Time Human Pose Recognition in Parts from Single Depth Images.” Presented at the IEEE Conference on Computer Vision and Pattern Recognition, USA.

[6] Kjærside, K., Kortbek, K. J., and Hedegaard, H. 2005. "ARDressCode: Augmented Dressing Room with Tag-Based Motion Tracking and Real-Time Clothes Simulation,” Presented at the Central European Multimedia and Virtual Reality Conference, Czech Republic.

[7] MakeHuman. 2014. "MakeHuman ${ }^{\mathrm{TM}}$ and Its Purpose." http://www.makehuman.org/doc/node/makehuman_and_i ts_purpose.html.

[8] Cho, H., and Schwarz, N. 2010. "I Like Those Glasses on You, But Not in the Mirror: Fluency, Preference, and Virtual.” Journal of Consumer Psychology 20: 471-5.

[9] Wang, L., Villamil, R., Samarasekera, S., and Kumar, R. 2012. "Magic Mirror: A Virtual Handbag Shopping System.” Presented at the 2012 IEEE Computer Society Conference on Computer Vision and Pattern Recognition Workshops, USA.

[10] Unity 3D. 2014. http://unity3d.com/unity. 